\section{Bei Weinüberempfindlichkeit gibt es viele Verdächtige}

\author{
Unverträglichkeitsreaktionen auf Wein können vielfältige Ursachen \\ haben. Unterschieden werden „echte“, IgE-vermittelte Allergien und \\ pseudoallergische Überempfindlichkeitsreaktionen - die Sympto- \\ matik kann aber bei beiden Formen gleich sein. Will man der Ursache \\ auf die Spur kommen, ist viel diagnostischer Spürsinn gefragt.
}

B ei einer „Weinüberempfindlichkeit“ müssen als Auslöser sowohl die Inhaltsstoffe der Weintraube als auch die während des Verarbeitungsprozesses hinzu gegebenen Hilfsmittel wie z. B. Hefen, Klärmittel und Schwefelverbindungen in Betracht gezogen werden. Außerdem seien bei den Weinproduzenten andere Reaktionen anzutreffen als bei den Weinkonsumenten, erklärte Priv.-Doz. Dr. Vera Mahler, Erlangen.

Überempfindlichkeiten im Zusammenhang mit dem Weinanbau und der Weinherstellung sind bei Winzern als Berufskrankheit anerkannt. Dazu gehören beispielsweise Typ-1-Allergien gegen Weinpollen. Wein ist zwar kein Windbestäuber, trotzdem können bei engem Kontakt mit der blühenden Weinpflanze Pollenallergien auftreten. Relativ häufig sind bei Winzern auch Atemwegsallergien gegen Schimmel und Spinnmilben - eine Spinnmilbenallergie kommt bei Winzern sogar häufiger vor als eine Hausstaubmilbenallergie. Schimmel (meist aus dem Weinkeller) kann Auslöser einer exogenen allergischen Alveolitis sein. Auch eine Sensibilisierung gegen das bei der Weinherstellung verwendete Schwefeldioxid ist eine Winzer-Berufskrankheit, Symptome sind hier meist Kontakturtikaria und Asthma.

\section{Triggerfaktoren erschweren Diagnostik}

Überempfindlichkeiten gegen Schwefeldioxid bzw. Sulfite sind auch beim Weinkonsumenten bekannt. Symptome sind Hautreaktionen, daneben Asthma oder eine Anaphylaxie. Die Symptome treten dosisabhängig auf, wobei beim Weißwein offensichtlich schon die Aufnahme von 1 bis $5 \mathrm{mg}$ klinische Erscheinungen aus- lösen kann, beim Rotwein sind es meist Mengen zwischen 20 bis $50 \mathrm{mg}$. Die $\mathrm{SO}_{2}$-Grenzwerte für Rotwein wurden in Deutschland auf $160 \mathrm{mg} / \mathrm{l}$ und beim Weißwein auf $210 \mathrm{mg} / \mathrm{l}$ festgelegt, moderate Trinkmengen dürften deshalb nur selten Reaktionen auslösen. Wie bei jeder Nahrungsmittelunverträglichkeit können aber Begleitfaktoren die Reaktionsschwelle herabsetzen. In Frage kommen dafür Inhaltsstoffe des Weins - der Patient mit $\mathrm{SO}_{2}$-Intoleranz verträgt dann z. B. nur eine bestimmte Weinsorte nicht - oder Begleitumstände wie körperliche Belastung, andere Nahrungsmittel oder Infektionserkrankungen

Überempfindlichkeiten werden vereinzelt auch beobachtet gegen Sorbinsäure sowie Salicylate, die im Weinbau zum

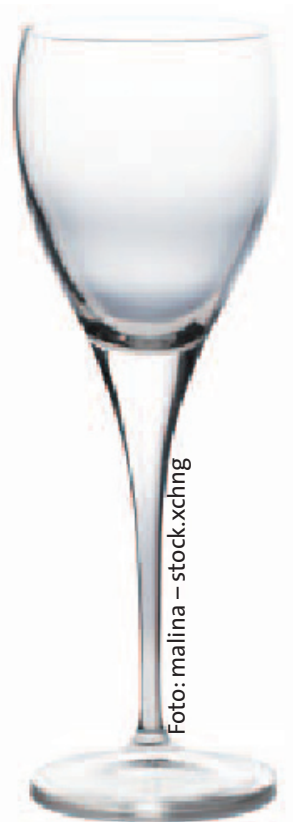

Karenz ist bei Weinallergie die einfachste Therapie - aber nicht jedermanns Sache.

\section{Histaminintoleranz}

Die Histaminintoleranz imponiert durch ein vielfältiges Symptombild: Neben Juckreiz, Urtikaria und Asthmaanfällen kommen auch Übelkeit und Diarrhö, Blutdruckabfall und Flush vor. Auslöser der Reaktionen ist ein Überangebot von Histamin in der Nahrung, das durch die histaminabbauende Diaminoxidase (DAO) in der Darmschleimhaut nicht mehr ausreichend abgebaut werden kann. Eine verminderte DAO-Produktion ist meist erworben und findet sich gehäuft z. B. bei Patienten mit chronischen entzündlichen Darmerkrankungen. Bei Zufuhr von „Histaminlockern“ wie Alkohol oder histaminreichen Nahrungsmitteln wie Fisch, Erdbeeren, Spinat oder Schokolade wird die Histaminabbaukapazität der DAO überschritten und die Symptomatik beginnnt.

Pflanzenschutz und zur Pflanzenstärkung eingesetzt werden. Eine Weinunverträglichkeit kann außerdem auf einer Histaminintoleranzreaktion beruhen und wird nach Rotwein häufiger als nach Weißwein gesehen. Getriggert werden die Symptomausbrüche auch bei dieser Unverträglichkeit durch Begleitfaktoren, infrage kommen z. B. eine Fischmahlzeit oder die Einnahme von Acetylsalicylsäure oder Metamizol. Eine Weinintoleranz aufgrund eines relativen Mangels des Alkohol-abbauenden Enzyms Acetaldehyd-Dehydrogenase schließlich kommt bei Asiaten gehäuft vor. In diesen Fällen resultiert u. a. eine Flush-Symptomatik.

\section{Typ-I-Allergien gegen Inhalts- und Zusatzstoffe}

Typ-I-Allergien gegen Wein beruhen auf Sensibilisierungen gegen Allergene aus den Weintrauben, die hauptsächlich aus der Gruppe der Lipidtransferproteine stammen. Diese Allergene rufen vor allem ein orales Allergiesymptom, Urtikaria und Asthma hervor. Die in der Weinverarbeitung zugesetzten Klärungsmittel liefern gleich mehrere Allergene. Zwar werden die Zusätze im Laufe des Fertigungsprozesses wieder entfernt, dennoch finden sich in einigen Weinen Gluten, Casein sowie Erbsen- oder Lupinenextrakte noch in relevanten Konzentrationen.

$b k$

Mahler V. Aktuelles zur Weinallergie 